\title{
Nonlinear Spectral-Spatial Control and Localization of Supercontinuum Radiation
}

\author{
Dragomir N. Neshev, ${ }^{1}$ Andrey A. Sukhorukov, ${ }^{1}$ Alexander Dreischuh, ${ }^{1,2}$ Robert Fischer, ${ }^{1}$ Sangwoo Ha, ${ }^{1}$ Jeremy Bolger, ${ }^{3}$ \\ Lam Bui, ${ }^{4}$ Wieslaw Krolikowski, ${ }^{1}$ Benjamin J. Eggleton, ${ }^{3}$ Arnan Mitchell, ${ }^{4}$ Michael W. Austin, ${ }^{4}$ and Yuri S. Kivshar ${ }^{1}$ \\ ${ }^{1}$ Centre for Ultra-high Bandwidth Devices for Optical Systems (CUDOS), Nonlinear Physics Centre and Laser Physics Centre, \\ Research School of Physical Science and Engineering, Australian National University, Canberra ACT 0200, Australia \\ ${ }^{2}$ Department of Quantum Electronics, Faculty of Physics, Sofia University, Bulgaria \\ ${ }^{3}$ School of Physics, University of Sydney, Sydney NSW 2006, Australia \\ ${ }^{4}$ School of Electrical and Computer Engineering, RMIT University, Melbourne, Australia
}

(Received 28 February 2007; published 18 September 2007)

\begin{abstract}
We present the first observation of spatiospectral control and localization of supercontinuum light through the nonlinear interaction of spectral components in extended periodic structures. We use an array of optical waveguides in a $\mathrm{LiNbO}_{3}$ crystal and employ the interplay between diffraction and nonlinearity to dynamically control the output spectrum of the supercontinuum radiation. This effect presents an efficient scheme for optically tunable spectral filtering of supercontinua.
\end{abstract}

DOI: 10.1103/PhysRevLett.99.123901

The generation of supercontinuum radiation is one of the most fascinating examples of extreme nonlinear processes where many colors of light are created from a narrow-band source [1-3]. Supercontinuum generation has found various applications in diverse fields of physics, including optical frequency metrology [4], spectroscopy [5], tomography [6], and optical characterization of photonic band gap materials [7]. Supercontinuum radiation differs significantly from the light emitted by incoherent light sources, combining high spatial coherence and spectral brightness. Furthermore, its high peak intensity and average power enables strong light-matter interactions in the nonlinear regime [8].

A lot of effort in recent years has been concentrated on the manipulation of temporal and spectral characteristics of supercontinuum generation in photonic crystal fibers (PCFs) [2]. PCFs allow for engineering of the spectral dispersion and confinement of light through the underlying periodicity of their structure. Periodic photonic structures also find applications for spatial beam control; however, their use is primarily optimized for beam shaping and deflection in a narrow-frequency range. Since the Bragg scattering in a periodic structure is dependent on the propagation direction and light wavelength, polychromatic beams will become spectrally separated inside the structure. This opens an intriguing physical problem of how to manipulate broadband beams in periodic structures. Furthermore, various applications would benefit from the ability to sort the ultrawide spectrum into individual spatial channels and perform dynamic modification of the supercontinuum spectrum. In this Letter, we demonstrate novel possibilities for all-optical spatial switching with simultaneous spectral reshaping and localization of supercontinuum light beams. We achieve this goal by employing the nonlinear interactions of multiple colors in an array of optical waveguides. We find that an interplay of wave scattering from a periodic structure and nonlinear selfaction allows one to separate selectively or combine differ-
PACS numbers: 42.65.Tg, 42.65.Jx, 42.65.Wi

ent spectral components. Additional flexibility is implemented through interaction with induced defects, where small refractive-index changes lead to strong beam reshaping and spectral filtering.

In our experiments, we use the supercontinuum light generated by femtosecond laser pulses (140 fs at $800 \mathrm{~nm}$ from a Ti:Sapphire oscillator) coupled into $1.5 \mathrm{~m}$ of highly nonlinear photonic-crystal fiber (Crystal Fiber NL-2.0-740 with engineered zero dispersion at $740 \mathrm{~nm}$ ). The spectrally narrow pump pulses are converted into the broad supercontinuum spectrum through several processes [1-3], including self-phase modulation, soliton formation due to the interplay between anomalous dispersion and Kerr nonlinearity, soliton breakup due to higher order dispersion, and Raman shifting of the solitons, leading to nonsolitonic radiation in the short-wavelength range. The spectrum of the generated supercontinuum measured by a fiber spectrometer is shown in Fig. 1(a), and it spans over the range of 450-850 nm. After recollimation, the supercontinuum beam is attenuated by neutral-density filters to control its total power while preserving its spectrum. The beam is then refocused by a microscope objective $(\times 20)$ to a

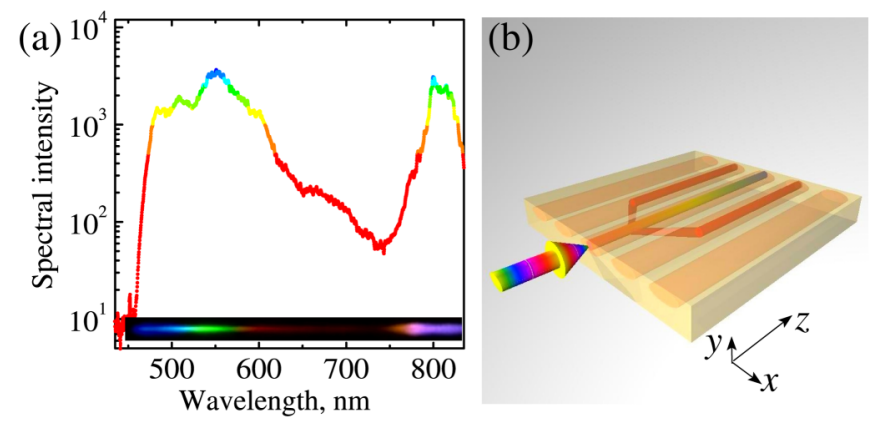

FIG. 1 (color online). (a) Spectrum of the generated supercontinuum radiation. (b) Schematic of the experiment illustrating the dispersion of the colors inside the array of optical waveguides. 
single channel of a waveguide array [Fig. 1(b)]. The optical waveguides are fabricated by indiffusion of a $100 \AA$ thin layer of Titanium in a X-cut, $50 \mathrm{~mm}$ long monocrystal lithium niobate wafer [9]. The waveguides are spaced at a period of $d=19 \mu \mathrm{m}$ and are single moded for all supercontinuum spectral components. An important property of the $\mathrm{LiNbO}_{3}$ crystal is that high laser powers induce nonlinear change of the optical refractive index due to photorefraction [10,11]. The photovoltaic nonlinearity [12] is of defocusing type, meaning that an increase of the light intensity leads to a decrease in the material refractive index. The output of the array is imaged by a microscope objective $(\times 5)$ onto a color CCD camera, where a dispersive $60^{\circ}$ (glass SF-11) prism could be inserted between the imaging objective and the camera to resolve all supercontinuum spectral components.

When the light propagates in the array, it couples between the neighboring waveguides experiencing discrete diffraction [13-15]. In sharp contrast to the diffraction in bulk materials, the process of discrete diffraction results in most of the light being directed into the wings of the beam. The light couples from one waveguide to another due to the spatial overlaps of the waveguide modes. Since the mode profile and confinement depend on the wavelength, the discrete diffraction exhibits strong spectral dispersion. The mode overlap at neighboring waveguides is much stronger for redshifted components, which therefore diffract faster than their blue counterparts. This leads to spatial redistribution of the colors of the supercontinuum radiation, and the red and blue components are transversely separated at the output. To gain deeper insight into the output spectral characteristics, we separate the individual colors in the vertical direction by a prism, see Fig. 2(a). This spectrally resolved discrete diffraction of the supercontinuum beam allows for the precise determination of the linear parameters of the periodic structure, realizing a natural application of the supercontinuum light for single shot characterization of periodic structures and photonic crystals [16].
The spectrally resolved diffraction patterns allows one to determine the dispersion of the coupling between the individual waveguides of the array $[17,18]$, characterized by the coupling coefficient, $C=\pi /\left(2 L_{c}\right)$, where $L_{c}$ is the characteristic distance for the light to couple from one waveguide to another. The evaluated wavelength dependence of $C$ in our experiments is presented in Fig. 2(b) (circles) together with the corresponding polynomial fit to the experimental data (solid line). The measured waveguide coupling is a monotonically increasing function of the wavelength with approximately 10 times difference for both ends of the supercontinuum spectrum. Knowing the characteristic parameters of the periodic structure, we can simulate the spectral field distribution inside the array by solving a set of equations for the spatial beam envelopes $A_{m}(x, z)$ of different frequency components at vacuum wavelengths $\lambda_{m}, \quad i \partial A_{m} / \partial z+\lambda_{m}\left(4 \pi n_{0}\right)^{-1} \partial^{2} A_{m} / \partial x^{2}+$ $2 \pi \lambda_{m}^{-1} \Delta n\left(x ; \lambda_{m}\right) A_{m}=0$, where $x$ and $z$ are the transverse and longitudinal coordinates, respectively, and $n_{0}=2.3$ is the average refractive index. We note that the dominant dispersion contribution comes from the geometric dispersion. Therefore, the value of $n_{0}$ can be taken as constant for the whole spectral range, while the effective refractiveindex modulation $\Delta n\left(x ; \lambda_{m}\right)$ exhibits significant dispersion. For the fabricated waveguide arrays away from the boundaries, the modulation can be accurately described as $\Delta n(x ; \lambda)=\Delta n_{\max }(\lambda) \cos ^{2}(\pi x / d)$, where the wavelength dependence of the effective modulation depth $\Delta n_{\max }(\lambda)$ is calculated by matching the experimentally measured waveguide coupling, as shown in Fig. 2(b). Numerical simulation of supercontinuum light diffraction in the waveguide array shows that the spectral components progressively become spatially separated along the propagation distance, see Fig. 2(c).

An important task for manipulation of broadband optical signals is the ability to tune the spectral transmission in the spatial domain. Here, we propose and demonstrate a novel approach to flexible spatial-spectral control of supercontinuum radiation realized through the effect of nonlinear
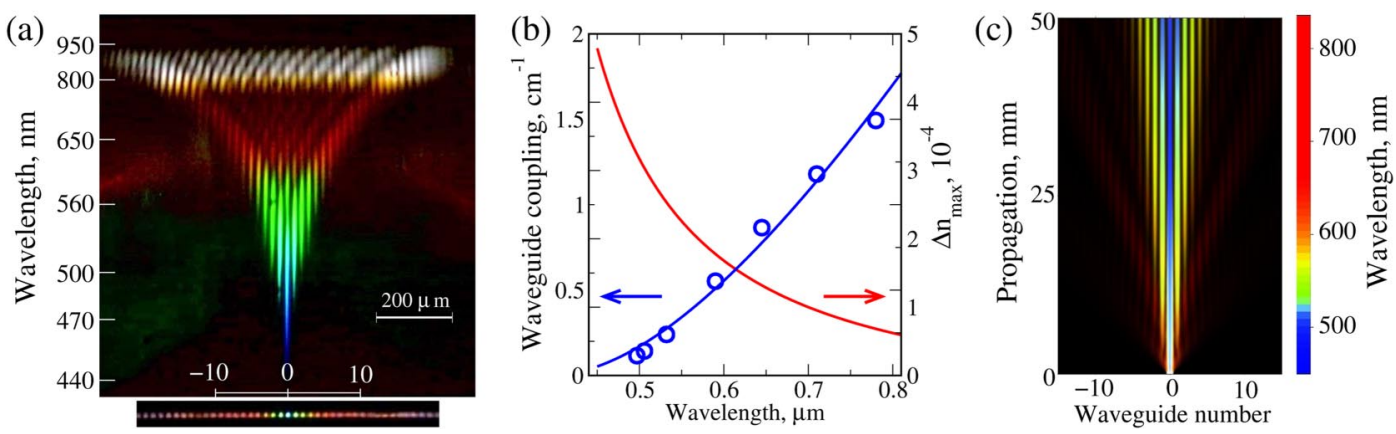

FIG. 2 (color online). (a) Spectrally resolved diffraction of the supercontinuum radiation (low input power of $17 \mu$ W). Bottom: output color intensity profile. (b) Measured wavelength dispersion of the waveguide coupling (circles), corresponding fit to the experimental data (solid line), and estimated dispersion of the refractive-index modulation (indicated by arrows). (c) Numerically calculated propagation of the supercontinuum inside the sample. In simulations, the input spatial profiles of all spectral components have Gaussian shape with full width at half-maximum of peak intensity (FWHM) of $7.5 \mu \mathrm{m}$. 
interaction and selective self-trapping of spectral components inside the individual channels of the array. As the supercontinuum radiation has high averaged intensity due to its high spatial coherence and small beam size down to a single waveguide of the array, it can change the material refractive index due to the photorefractive response of the $\mathrm{LiNbO}_{3}$ crystal. The optically-induced refractive-index change is defined by the time-averaged light intensity of different spectral components $[19,20]$ due to the slow time response of the photorefractive nonlinearity. As a result, the total spectral power distribution is preserved, allowing one to perform spatial switching and reshaping of polychromatic signals without generating or depleting different spectral regions as in the process of four-wave mixing. This provides a fundamentally different regime compared to dynamics of light with supercontinuum spectrum in multicore photonic-crystal fibers with fast nonlinear response [21,22], where nonlinearly-induced spatial mode reshaping is inherently accompanied by the spectral transformations.

We observe that as the laser power is increased, the beam begins to localize, bringing more and more wavelength components into the central waveguide. This process is characterized by a threshold wavelength. Spectral components below this threshold are trapped in the central waveguide, while longer wavelength components are delocalized. A representative example is shown in Fig. 3(a), where all spectral components below $600 \mathrm{~nm}$ are trapped to a single waveguide for supercontinuum power of $7.5 \mathrm{~mW}$. Whereas a defocusing nonlinearity would result in accelerated beam spreading in bulk nonlinear media, it can support beam localization and formation of monochromatic [9,23-27] and polychromatic [28] spatial gap solitons. This is the case as the defocusing nonlinearity can balance the anomalous beam diffraction appearing due to Bragg scattering from the periodic waveguide array, representing a different physical picture compared to the theoretically studied white-light solitons in lattices supported by focusing nonlinear response [29].

The important characteristic of this localization process is that it combines all wavelength components of the super- continuum spectrum, and therefore the wavelength dependent spatial waveguide dispersion is strongly suppressed in the nonlinear regime. This is illustrated in Fig. 3(b), which shows the amount of spectral power trapped into the central waveguide for five different wavelengths. Our result demonstrates that the localization happens at lowest powers for the blue spectral components $(470 \mathrm{~nm})$, which experience weakest diffraction. The threshold localization power is higher at longer wavelengths $(500,580,645$, and $780 \mathrm{~nm})$ as stronger nonlinearity is necessary to balance the stronger diffraction at these wavelengths.

To achieve a better understanding of the localization process inside the array, we model the nonlinear propagation and interaction of all spectral components, by including a nonlinear spectral response into the refractive-index modulation $\Delta n$. We take the nonlinear detuning in the form $\Delta n_{\mathrm{nl}}(x, z ; \lambda)=\gamma M^{-1} \sum_{j=1}^{M} \sigma\left(\lambda_{j}\right)\left|A_{j}\right|^{2}$, which accounts for photovoltaic defocusing nonlinearity $[12,20]$ in the regime of weak saturation, where $\gamma$ is the nonlinear coefficient $(\gamma<0$ for defocusing nonlinearity). We approximate the photosensitivity dependence in $\mathrm{LiNbO}_{3}$ waveguides by a Gaussian function $\sigma(\lambda)=\exp [-\log (2)(\lambda-$ $\left.\left.\lambda_{b}\right)^{2} / \lambda_{w}^{2}\right]$ with $\lambda>\lambda_{b}=400 \mathrm{~nm}$ and $\lambda_{w}=150 \mathrm{~nm}$, and choose the number of frequency components $M=50$. The results of the numerical simulations are shown in Fig. 3(c) for a power of $7.5 \mathrm{~mW}$, where the stationary beam selftrapping is established after the first $10 \mathrm{~mm}$ of propagation inside the sample. We also calculate the relative spectral powers in the central waveguide and find good agreement with the experimental results, see Fig. 3(b).

A specific characteristic of the localization process in $\mathrm{LiNbO}_{3}$ is the nonlinear response time. It is inversely proportional to the input laser power, and it can vary from a few seconds to several minutes at low light intensities [30]. The advantage of this slow response is that once the refractive-index modulation is written, it can be preserved in the structure for a long period of time, provided the sample is not exposed to strong light illumination [10]. This opens a novel possibility for dynamic writing of defects with arbitrary geometry $[11,31,32]$. We demon-
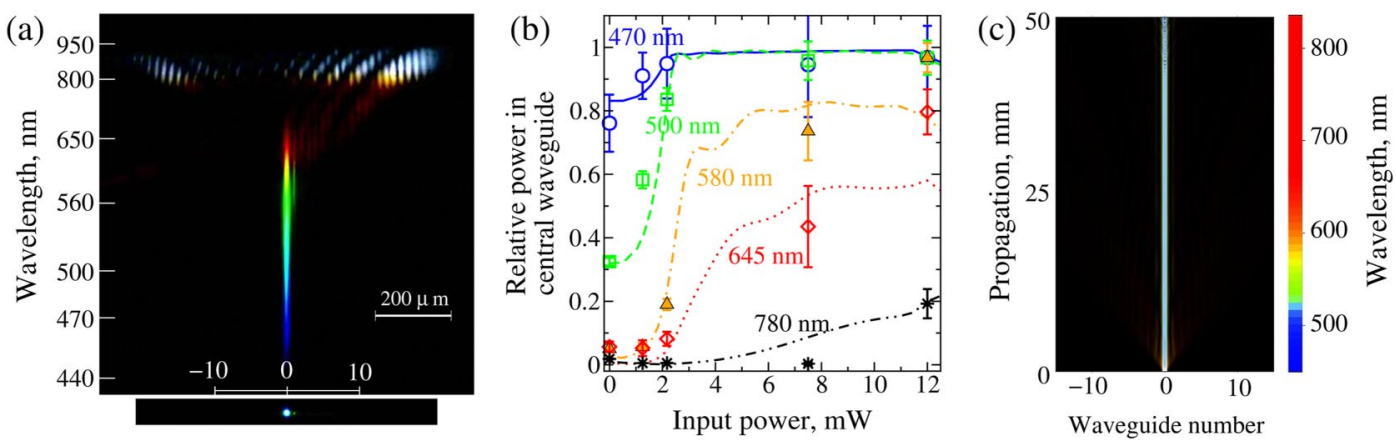

FIG. 3 (color online). (a) Spectrally resolved localization of the supercontinuum inside the central waveguide at input power of $P_{\text {in }}=7.5 \mathrm{~mW}$. Bottom: output intensity distribution. (b) Measured (points) and calculated (lines) relative spectral power in the central waveguide as a function of the input power for five different spectral components, as indicated by labels. (c) Numerically calculated nonlinear propagation at $P_{\text {in }} \simeq 7.5 \mathrm{~mW}$. In simulations, dimensionless power $P_{0}=M^{-1} \sum_{j=1}^{M} \int\left|A_{j}\right|^{2} d x$ is normalized to $1 \mathrm{~mW}$ and $\gamma \simeq-1.28 \times 10^{-4}$. 


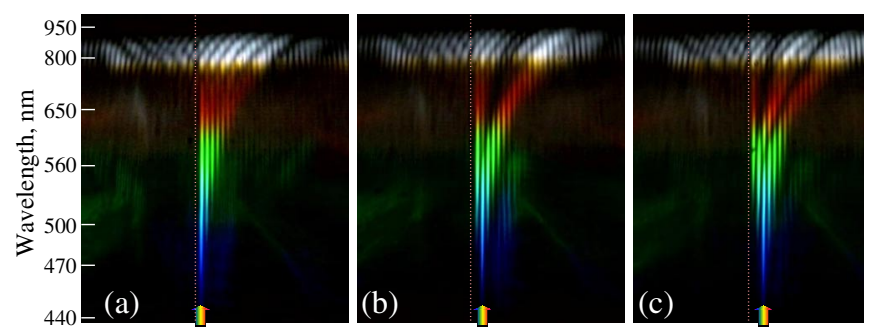

FIG. 4 (color online). Reflection and tunneling of spectral components from an optically-induced defect. The array is probed (a) next to the defect; (b) one waveguide away; (c) two waveguides away.

strate that defects can act as spectral filters for the supercontinuum light. In experiment, we generate a localized supercontinuum state at power of $12 \mathrm{~mW}$. Several hours after switching off the writing beam, we probe the induced defect with a low power supercontinuum beam. When the light is injected into the waveguide adjacent to the defect [Fig. 4(a)], we observe reflection of all spectral components below a threshold wavelength value (approximately $800 \mathrm{~nm}$ in our case). On the other hand, spectral components with longer wavelengths are transmitted to the lefthand side of the defect. When the input beam is injected into the second or third waveguide away from the defect, we observe complex spectral reshaping of the output transmission due to reflection of the supercontinuum from the defect state. We note that the nonlinear refractive-index modulation in the detuned waveguide is only of the order of $10^{-4}$, but it is sufficient to modify significantly the spectrum of the supercontinuum radiation. This effect is somehow similar to surface manipulation of the supercontinuum light [33], but with the ability for reconfiguration and tuning of the defect properties. Similar functionality can also be implemented by electro-optic tuning of individual waveguides of the array.

The spectral filtering by the defect can be approximately described using the discrete model approach [15], $i d \psi_{n}\left(\lambda_{m}\right) / d z+C\left(\lambda_{m}\right)\left[\psi_{n-1}\left(\lambda_{m}\right)+\psi_{n+1}\left(\lambda_{m}\right)\right]-\lambda_{m}^{-1} \rho_{n}=$ 0 , where $\psi_{n}\left(\lambda_{m}\right)$ are the mode amplitudes at the waveguide number $n$, and $\rho_{n}$ defines the effective strength of the induced defect. For the defect induced at a single site, $\left|\rho_{n}\right| \ll\left|\rho_{0}\right|$ at $n \neq 0$. Then, the power transmission through the defect is limited from above by $T\left(\lambda_{m}\right)=\{1+$ $\left.\rho_{0}^{2}\left[2 C\left(\lambda_{m}\right) \lambda_{m}\right]^{-2}\right\}^{-1}$. Since the coupling $C(\lambda)$ increases at longer wavelengths, the transmission coefficient $T(\lambda)$ grows monotonously, providing a mechanism for selective reflection of spectral components where the cutoff wavelength can be tuned by adjusting the defect strength.

In conclusion, we have presented the first observation of all optically tunable spatiospectral reshaping of supercontinuum light in nonlinear periodic photonic structures. We demonstrated that spatially separated spectral components can be trapped together by nonlinearity induced index change in the material, with a wavelength threshold proportional to the total beam power. We also expect that the demonstrated effects can be useful for nonlinear control of wavelength dispersion of ultrabroad spectrum pulses, offering additional functionality for dispersion compensation in fiber lasers.

[1] J. K. Ranka, R. S. Windeler, and A. J. Stentz, Opt. Lett. 25, 25 (2000).

[2] P. St. J. Russell, Science 299, 358 (2003).

[3] J. M. Dudley, G. Genty, and S. Coen, Rev. Mod. Phys. 78, 1135 (2006).

[4] T. Udem, R. Holzwarth, and T. W. Hansch, Nature (London) 416, 233 (2002).

[5] A. M. Zheltikov, Usp. Fiz. Nauk 176, 623 (2006) [Phys. Usp. 49, 605 (2006)].

[6] B. Povazay et al., Opt. Lett. 27, 1800 (2002).

[7] M. H. Qi et al., Nature (London) 429, 538 (2004).

[8] M. Balu, J. Hales, D. J. Hagan, and E. W. Van Stryland, Opt. Express 13, 3594 (2005).

[9] M. Matuszewski et al., Opt. Express 14, 254 (2006).

[10] A. Ashkin et al., Appl. Phys. Lett. 9, 72 (1966).

[11] D. Kip, Appl. Phys. B 67, 131 (1998).

[12] G. C. Valley et al., Phys. Rev. A 50, R4457 (1994).

[13] A. L. Jones, J. Opt. Soc. Am. 55, 261 (1965).

[14] S. Somekh, E. Garmire, A. Yariv, H. L. Garvin, and R. G. Hunsperger, Appl. Phys. Lett. 22, 46 (1973).

[15] D. N. Christodoulides, F. Lederer, and Y. Silberberg, Nature (London) 424, 817 (2003).

[16] P. T. Rakich et al., Opt. Express 13, 821 (2005).

[17] R. Iwanow et al., Phys. Rev. Lett. 95, 053902 (2005).

[18] A. Szameit et al., Appl. Phys. B 82, 507 (2006).

[19] M. Mitchell and M. Segev, Nature (London) 387, 880 (1997).

[20] H. Buljan, T. Schwartz, M. Segev, M. Soljacic, and D. N. Christodoulides, J. Opt. Soc. Am. B 21, 397 (2004).

[21] D. A. Akimov et al., Appl. Phys. B 77, 299 (2003).

[22] A. Betlej et al., Opt. Lett. 31, 1480 (2006).

[23] Yu. S. Kivshar, Opt. Lett. 18, 1147 (1993).

[24] J. Feng, Opt. Lett. 18, 1302 (1993).

[25] R. F. Nabiev, P. Yeh, and D. Botez, Opt. Lett. 18, 1612 (1993).

[26] J. W. Fleischer, M. Segev, N. K. Efremidis, and D. N. Christodoulides, Nature (London) 422, 147 (2003).

[27] F. Chen et al., Opt. Express 13, 4314 (2005).

[28] K. Motzek, A. A. Sukhorukov, and Yu. S. Kivshar, Opt. Express 14, 9873 (2006).

[29] R. Pezer, H. Buljan, G. Bartal, M. Segev, and J. W. Fleischer, Phys. Rev. E 73, 056608 (2006).

[30] P. Gunter, Phys. Rep. 93, 199 (1982).

[31] A. Guo, M. Henry, G. J. Salamo, M. Segev, and G. L. Wood, Opt. Lett. 26, 1274 (2001).

[32] P. Zhang, D. X. Yang, J. L. Zhao, and M. R. Wang, Opt. Eng. (Bellingham, Wash.) 45, 074603 (2006).

[33] A. A. Sukhorukov et al., Opt. Express 14, 11265 (2006). 